Hautarzt 2018 69 (Suppl 1):S1

https://doi.org/10.1007/s00105-018-4216-4

(c) Springer Medizin Verlag GmbH, ein Teil von Springer Nature 2018

CrossMark

Tanja v. Braunmühl · Michael J. Flaig · llana Goldscheider · Hans Wolff · Thomas Herzinger $\cdot$ Thomas Ruzicka

Klinik und Poliklinik für Dermatologie und Allergologie, Ludwig-Maximilians-Universität, München, Deutschland

\title{
Vorwort zur DIA-KLINIK der 26. Fortbildungswoche für praktische Dermatologie und Venerologie
}

Auch in diesem Jahr können wir Ihnen wieder ein großes Spektrum dermatologischer Fälle präsentieren, die von komplexen Autoimmundermatosen bis hin zu onkologischen „Wunderheilungen" reichen. In einigen Fällen demonstrieren wir, dass „das Häufige häufig ist", während wir in anderen Kasuistiken genau diesen Leitsatz dann widerlegen müssen.

Nicht nur die mündliche Präsentation der Kasuistiken im Rahmen der Fortbildungswoche, sondern auch die schriftliche Publikation mit zahlreichen Abbildungen ist hervorzuheben. Die gebündelte Sammlung der Fälle in einer Printausgabe lädt zum Mitblättern und auch Nachschlagen ein.

Wir freuen uns, dass wir Ihnen dieses Jahr erstmals unsere Fälle im Rahmen eines Sonderheftes der Zeitschrift Der Hautarzt präsentieren können, und bedanken uns für die freundliche Unterstützung durch LEO Pharma GmbH.

Tanja v. Braunmühl

Michael J. Flaig

Ilana Goldscheider

Hans Wolff

Thomas Herzinger

Thomas Ruzicka

\section{Korrespondenzadresse}

PD Dr. T. v. Braunmühl

Klinik und Poliklinik für Dermatologie und Allergologie, Ludwig-Maximilians-Universität Frauenlobstr. 9-11, 80337 München,

Deutschland

Tanja.vonBraunmuehl@med.uni-muenchen.de

Interessenkonflikt. T.v. Braunmühl, M.J. Flaig, I. Goldscheider, H. Wolff, T. Herzinger und T. Ruzicka geben an, dass kein Interessenkonflikt besteht.

This article is part of a supplement sponsored by LEO Pharma GmbH. 